\title{
Multiple Neuroinvasive Pathways in COVID-19
}

\author{
Dmitri Bougakov $^{1}$ (I) $\cdot$ Kenneth Podell ${ }^{2} \cdot$ Elkhonon Goldberg $^{1,3}$
}

Received: 8 July 2020 / Accepted: 25 September 2020 / Published online: 29 September 2020

(C) Springer Science+Business Media, LLC, part of Springer Nature 2020

\begin{abstract}
COVID-19 is a highly infectious viral disease caused by the novel coronavirus SARS-CoV-2. While it was initially regarded as a strictly respiratory illness, the impact of COVID-19 on multiple organs is increasingly recognized. The brain is among the targets of COVID-19, and it can be impacted in multiple ways, both directly and indirectly. Direct brain infection by SARS-CoV-2 may occur via axonal transport via the olfactory nerve, eventually infecting the olfactory cortex and other structures in the temporal lobe, and potentially the brain stem. A hematogenous route, which involves viral crossing of blood-brain barrier, is also possible. Secondary mechanisms involve hypoxia due to respiratory failure, as well as aberrant immune response leading to various forms of encephalopathy, white matter damage, and abnormal blood clotting resulting in stroke. Multiple neurological symptoms of COVID-19 have been described. These involve anosmia/ageusia, headaches, seizures, mental confusion and delirium, and coma. There is a growing concern that in a number of patients, long-term or perhaps even permanent cognitive impairment will persist well after the recovery from acute illness. Furthermore, COVID-19 survivors may be at increased risk for developing neurodegenerative diseases years or decades later. Since COVID-19 is a new disease, it will take months or even years to characterize the exact nature, scope, and temporal extent of its long-term neurocognitive sequelae. To that end, rigorous and systematic longitudinal follow-up will be required. For this effort to succeed, appropriate protocols and patient registries should be developed and put in place without delay now.
\end{abstract}

Keywords COVID-19 $\cdot$ NeuroCovid $\cdot$ Encephalopathy $\cdot$ Cognitive impairment $\cdot$ Neurocognitive sequelae

\section{The Current COVID-19 Pandemic}

The current COVID-19 pandemic is caused by severe acute respiratory syndrome coronavirus 2 (SARS-CoV-2). Based on World Health Organization definitions, the disease is named coronavirus disease - COVID-19; however, the virus causing the disease is severe acute respiratory syndrome coronavirus 2-SARS-CoV-2. The disease was first identified in Wuhan, China at the end of 2019, and has rapidly spread throughout the world resulting in a global pandemic [1]. Based on Johns Hopkins University Coronavirus Resource Center, as of the second week of June 2020, 188 countries and regions have been affected, and more and 7 million people were infected, with more than 400,000 deaths [2]. Unfortunately, these numbers continue to grow.

Elkhonon Goldberg

eg@elkhonongoldberg.com

Luria Neuroscience Institute, New York, NY, USA

2 Houston Methodist Hospital and Weill Cornell Medical College, Houston, TX, USA

3 NYU Grossman School of Medicine, New York, NY, USA
Our current knowledge of the novel COVID-19 disease is limited, but new findings are accumulating rapidly. Initially, it was thought to be strictly an upper respiratory disease (hence its categorization as a SARS virus); however, as more details emerge, COVID-19 clearly can have both direct and secondary impact on the brain. In this article, we attempt to summarize what has been learned so far about the effect of COVID19 on the brain. This knowledge will undoubtedly expand very rapidly, and new findings and theories will emerge. Nevertheless, the sheer magnitude of the current impact of this pandemic justifies surveying the current knowledge in face of the limitations of an incomplete and rapidly evolving disease picture. We hope that this review will aid in the future research, diagnosis, and treatment of the neurological aspects of the COVID-19.

\section{Coronaviruses}

Coronaviruses are a group of related viruses found in mammals, including humans and bats, and birds. The name coronavirus originated from the visualized findings of spike-like glycoproteins on the surface membrane of the virus making it 
appear to have a crown ("corona" in Latin) [3]. The disease that they cause may range from asymptomatic/mild to lethal. Based on the Center for Disease Control (CDC) [4], seven strains of coronaviruses affecting humans have been identified, four of them leading to mostly mild illness, and three leading to severe, potentially lethal illness. The following coronaviruses usually lead to mild, cold-like illness in humans: 229E; NL63; OC43; and HKU1; however, a wide spectrum of disease presentation, including a severe disease course, has been known to occur in children [5]. The following coronaviruses can lead to severe illness primarily in adult humans: severe acute respiratory syndrome coronavirus (SARS-CoV); Middle East respiratory syndrome coronavirus (MERS-CoV); and severe acute respiratory syndrome coronavirus 2 (SARS-CoV-2).

\section{Cellular Mechanisms of Infection and Their Implications}

The spike glycoproteins serve a crucial role in viral binding to cellular receptors, more specifically to angiotensin-conversion enzyme 2 (ACE2). ACE2 is quite ubiquitous in the organism and serves multiple physiological roles, including blood pressure control and inflammation. SARS-CoV-2 has a high affinity to ACE2 receptors which are present in bronchial epithelial cells, endothelial cells, and neurons and on the surface of cells in multiple organs and anatomical locations, e.g., nasal, cavity, lungs, heart, kidneys, and intestines. SARS-CoV-2 attacks the organism by attaching itself to ACE2 via its spike protein, which allows its RNA to enter the healthy cell permitting the virus to replicate through a complex sequence of steps $[6,7]$. The ubiquity of ACE2 in multiple organs may account for the multiplicity and heterogeneity of COVID-19 symptoms. Based on animal models, all coronaviruses including human (e.g., SARS-CoV) and animal (e.g., bat coronavirus RaTG13) require ACE2 as the cell entry receptor by binding $\mathrm{CoV}$ spike glycoprotein to cell membranes [8]. SARSCov-2 has a particularly strong affinity for ACE2 $[9,10]$. ACE2 is also expressed in the nasal cavity epithelia, and it has been hypothesized that olfactory epithelium is a common early infection site. According to this hypothesis, SARS-CoV2 then enters the brain via the olfactory nerve and olfactory bulb [11]. It has been also reported that ACE2 is expressed in multiple brain structures. These include the brainstem, cortex, striatum, and hypothalamus [12-14]. Furthermore, ACE2 is expressed both in neurons and in glial cells throughout the brain, which makes both types of cells potentially vulnerable to the virus [14]. It has been suggested that ACE2 may influence GABA (gamma-aminobutyric acid) neurotransmission in the amygdala and possibly elsewhere in the brain, pointing to another possible target of the virus [15]. It has also been hypothesized that since ACE2 and nicotinic receptors co- express, smokers, and perhaps even former smokers, may be at increased risk for severe COVID-19 [16]. Since ACE2 is present in the brain, it is possible that SARS-CoV-2 can attack multiple structures in the brain directly. A similar virus, SARS-CoV, is capable of infecting the brain directly, particularly the brainstem [17]. Other coronaviruses (e.g., SARS, MERS) also impact the brain and peripheral nervous system [3, 18-24]. However, since SARS-CoV-2 has a higher ACE2 binding affinity than SARS-COV, it may have a higher potential for attacking the brain directly [8]. It should be noted, however, that the level of ACE2 expression in the brain is lower than in other organs, and additional receptors may play a role in SARS-CoV-2 invasion of the brain [25]. CD147 (also known as basigin) has been implicated as another receptor to which the SAR-CoV-2 spike protein is capable of binding, thus serving as an additional route of brain infection [26].

\section{COVID-19 Symptoms}

Initially, COVID-19 was predominantly characterized as a respiratory illness. The Center for Disease Control (CDC) lists the following common symptoms: fever, cough, shortness of breath or difficulty breathing, chills, repeated shaking with chills, muscle pain, headache, sore throat, and (more recently added) acute loss of taste or/and smell [27]. The question has been asked, whether the ageusia and/or anosmia is a primary neurological symptom or neurological consequence secondary to a respiratory illness. At least one case study suggests that these symptoms have a primary neurological cause [28]. As our understanding of COVID-19 evolved, it has become increasingly clear that multiple organs are impacted in COVID-19 directly including the lungs, heart, vascular system, digestive system, and brain. Furthermore, as the heterogeneity of COVID-19 symptoms has been increasingly recognized, not all of them respiratory, more and more neurological symptoms have been documented.

\section{Direct Infection Vs. Secondary Effects of COVID-19 in the Brain}

The effect of COVID-19 on the brain may take several forms, some via direct infection and others via secondary mechanisms, e.g., immune response or respiratory failure-induced hypoxia. Direct presence of the SARS-CoV-2 in the brain has been demonstrated through detection of SARS-CoV-2 RNA in the cerebrospinal fluid of infected patients [29]. It has been suggested that direct invasion of the central nervous system by the SARS-CoV-2 may occur by at least two routes: hematogenous and via axonal transport through certain cranial nerves, e.g., olfactory nerve [28]. The proposed route of axonal transport through cranial nerves consists of the following 
anatomical structures: nasal cavity $>$ olfactory nerve $>$ olfactory bulb $>$ piriform cortex $>$ brainstem, with nasal cavity being the origin and the brainstem being the terminus [30]. Interestingly, this route of brain infection has been demonstrated for the OC43 strain of coronavirus [31].

To the extent that SARS-CoV-2 can affect the brainstem, it has been hypothesized that the respiratory breakdown in COVID-19 patients may be at least in part caused by SARSCoV-2 infecting respiratory centers in the medulla oblongata and the pons [32]. Similar spread via synapse-connected route into the brainstem cardiorespiratory centers of the medulla oblongata has been demonstrated for other coronaviruses, thus increasing the likelihood of the same for SARS-CoV-2 [17]. This potentially has significant implications for medical management of respiratory illness [33, 34].

Evidence is mounting that SARS-CoV-2 may directly impact the gray matter of the brain [35]. The brains of deceased patients showed evidence of edema and partial neuronal degeneration. The white matter in the brain and the spine may also be affected by demyelinating lesions in COVID-19 [36]. According to the neuroimaging studies conducted by Turkish scientists, out of all COVID-19-infected patients in the intensive care unit with neurologic symptoms, 44\% (12/27) of patients had abnormal MRI findings. A cortical FLAIR signal abnormality was present in 10/27 (37\%) patients, whereas subcortical and deep white matter signal abnormalities on FLAIR images were present in 3 patients. Abnormal findings involved the frontal lobe in 4 , parietal lobe in 3 , occipital lobe in 4 , temporal lobe in 1 , insular cortex in 3 , and cingulate gyrus in 3 cases [37]. Regardless of whether the brain is compromised via primary or secondary pathway by SARS-CoV-2, current studies suggest that neurological complications in COVID-19 are associated with a relatively greater disease severity and potential mortality [38].

\section{Neurological Manifestations of COVID-19}

Multiple studies documented and characterized a variety of COVID-19-related pathological processes and neurological symptoms. A wide range of neurological symptoms were documented in a sample of COVID-19 patients in Wuhan, China (where the pandemic originated) [39]. These included loss of smell and taste; headaches; dizziness; impaired level of consciousness; ataxia; seizures; and acute cerebrovascular illness (ischemic and hemorrhagic CVAs). Headaches and dizziness were common symptoms ( $17 \%$ and $13 \%$, respectively). Overall, the neurological symptoms were present in $36.4 \%$ of the patient sample and tended to occur early in the disease state. The same group of scientists reported that certain neurological symptoms were more frequent among severe cases (45.5\%) than among non-severe cases $(30.2 \%)$. These symptoms included acute cerebrovascular illness ( $5.7 \%$ vs. $0.8 \%)$, impaired consciousness ( $14.8 \%$ vs. $2.4 \%$ ), and skeletal muscle injury (19.3\% vs. $4.8 \%$ ) [39].

Some of the earliest research on COVID-19 emerged from China where the pandemic started. However, as the pandemic spread, scientists from multiple countries around the world proceeded to contribute to this body of knowledge. A group of scientists from the UK reported impaired consciousness, acute cerebrovascular events, and muscle disease in COVID19-infected patients. These neurologic abnormalities were seen in up to $50 \%$ of the most severe cases [40].

French scientists reported that in a sample of 58 patients, $84 \%$ had neurological symptoms. These included encephalopathy, corticospinal tract dysfunction, agitation, and delirium. Neuroimaging studies revealed bilateral frontotemporal hypoperfusion and acute ischemic CVAs [41]. In another study, Turkish scientists reported that in a sample of 235 ICU patients, $50(21.3 \%)$ developed neurological symptoms [37].

A group of Spanish researchers reviewed the clinical histories in a large number of hospitalized patients diagnosed with COVID-19 in March 2020 [42]. Out of 841 patients, $57.4 \%$ developed some form of neurological symptom. The nonspecific symptoms including myalgias (17.2\%), headache $(14.1 \%)$, and dizziness $(6.1 \%)$ were present mostly in the early stages of infection. Anosmia (4.9\%) and dysgeusia (6.2\%) reportedly occurred mostly early and were more frequent in less severe cases. Anosmia and dysgeusia were the first clinical manifestation in $60 \%$ of the cases. Disorders of consciousness were also reported. They occurred in $19.6 \%$ of the cases and tended to manifest mostly in older patients and in severe and advanced cases. The following symptoms and their respective frequencies were also reported: myopathy (3.1\%), dysautonomia $(2.5 \%)$, cerebrovascular diseases $(1.7 \%)$, seizures $(0.7 \%)$, movement disorders $(0.7 \%)$, encephalitis $(n=$ 1), Guillain-Barré syndrome $(n=1)$, and optic neuritis $(n=1)$. Neurological complications were the main cause of mortality in $4.1 \%$ of the deceased study subjects.

A comprehensive review conducted by an international group of scientists representing the World Federation of Neurology compiled an impressive body of evidence that infection with SARS-CoV-2 affects the central nervous system (CNS), the peripheral nervous system (PNS), and muscles [43]. They indicated that headaches and alteration of voluntary and involuntary psychomotor activity are likely to be initial indicators of potential neurological involvement. Symptoms of anosmia, hyposmia, hypogeusia, and dysgeusia were reported as frequent early symptoms of coronavirus infection. The authors hypothesized that respiratory failure in COVID-19 is probably neurogenic in origin. The proposed mechanism was that of viral invasion of olfactory nerve with subsequent progression into rhinencephalon and respiratory centers of the brainstem. Additionally, incidents of largevessel ischemic strokes, cerebral venous thrombosis, and intracerebral and subarachnoid hemorrhages were hypothesized 
to be related to viral attachment to ACE2 receptors in blood vessel endothelium causing endotheliitis, coagulopathy, and arterial and venous thromboses. Acute hemorrhagic necrotizing encephalopathy was hypothesized to be related to cytokine storm. Incidents of frontal hypoperfusion, seizures, encephalopathy, meningitis, encephalitis, and myelitis were reported. While less frequent, neuromuscular syndromes were also reported. These included Guillain-Barre syndrome, Miller Fisher syndrome, polyneuritis cranialis, and viral myopathy with rhabdomyolysis. In conclusion, the group called for a creation of an international neuroepidemiological collaboration to help fight the current pandemic and its neurological sequelae [43].

Headache is among the most common neurological manifestations of COVID-19. Based on meta-analysis, headaches had been reported in 11-13\% of hospitalized, and in 6-10\% of symptomatic patients. It is usually a moderate-severe, bilateral headache with pulsating or pressing quality in the temporoparietal, forehead, or periorbital regions. It is typically characterized by a "sudden to gradual onset and poor response to common analgesics. The possible pathophysiological mechanisms of headache include activation of peripheral trigeminal nerve endings by the SARS-CoV2 directly or through the vasculopathy and/or increased circulating pro-inflammatory cytokines and hypoxia." [44]

A pair of pathophysiologically related neurological symptoms anosmia (loss of smell) and ageusia (loss of taste) are increasingly recognized as common in COVID-19. The onset of these symptoms is often sudden and initially they were thought to be secondary to respiratory illness. However, viral invasion of the olfactory bulb with subsequent CNS invasion was postulated to be a possible underlying mechanism, which potentially can explain why these symptoms often appear prior to the development of significant respiratory symptoms. In a large multicenter European sample of patients with mild to moderate COVID-19, $85.6 \%$ and $88.8 \%$ of the cases had olfactory and gustatory dysfunction, respectively [45]. Olfactory dysfunction was largely self-limiting; but gustatory dysfunction persisted even after the resolution of respiratory symptoms in more than $70 \%$ of the cases. In this study, too, anosmia and ageusia were sometimes the first symptoms, appearing before the respiratory symptoms [39]. Anosmia was the initial presentation in $36 \%$ patients in a COVID-19 sample in Spain [38]. In an effort to understand the mechanisms of anosmia, a team of European scientists observed two groups of COVID-19 patients with complete anosmia: those with and those without nasal obstruction. They found no significant association between loss of smell and the symptoms of nasal obstruction, rhinorrhea, or postnasal drip in the entire group with anosmia or hyposmia, and concluded that anosmia was not secondary to rhinal obstruction, but was a primary neurological symptom. Higher prevalence of self-reported smell and taste dysfunction was observed in this sample than previously reported. It was concluded that many cases of anosmia are not secondary to nasal obstruction or inflammation. Rather, a direct "viral spread through the olfactory neuroepithelium and invasion of the olfactory bulb and central nervous system" was the cause [46]. The case report by Politi et al. showing brain MRI reversible inflammatory changes in the olfactory bulb, tract, and gyrus rectus in a recovered COVID-19 patient is consistent with this conclusion [28].

Delirium, one of the less specific neurological symptoms, is not uncommon in COVID-19 patients, especially in patients on ventilators [47]. Its development can be related to both primary and secondary COVID-19 effects on the brain. A case of encephalitis with delirium in a COVID-19 patient has also been reported in Wuhan, China [48]. In one of the representative cases, a patient in Japan developed seizures and impaired consciousness [49]. On hospital admission, his Glasgow Coma Scale was 6. MRI showed ventriculitis and encephalitis, mostly in the right mesial temporal regions, affecting the hippocampus. Specific SARS-CoV-2 RNA was detected in the patient's CSF [49]. The hippocampal involvement should not be surprising, since the allocortex of the hippocampus is phylogenetically similar to the olfactory cortex/ piriform cortex [50] that primarily serves the olfactory function and these regions are anatomically connected [51].

Seizures have also been reported in COVID-19 patients. Multiple seizures, including generalized tonic-clonic, were reported in a 30-year-old female in Iran [52]. The relatively young age of the patient challenges the notion that young people do not develop severe forms of COVID-19. Additionally, concern has been raised that COVID-19 infection in epilepsy patients may increase the likelihood of seizures due to fever [53]. Severe hypoxia frequently occurs in COVID-19 patients due to respiratory failure/distress, putting them at risk for hypoxic/anoxic-related encephalopathy [54].

COVID-19 patients were shown to be at risk for acute ischemic and hemorrhagic cerebrovascular events of CVAs [55]. In a sample of 221 patients, $6 \%$ developed an ischemic CVA, hemorrhage, or cerebral vein thrombosis, with significant lethality [56]. Blood coagulation is often impaired in COVID-19, resulting in hypercoagulation, that can lead to arterial and venous thromboses, causing an ischemic CVA [57].

A study conducted in the UK revealed a significant rate of ischemic CVAs in older patients with COVID-19. A number of CVAs due to large vessel occlusions (both arterial and venous), sometimes in multiple territories, were reported in COVID-19 patients in their 50s-80s [57]. In a different study, a surge of CVAs due to large vessel occlusions was reported in COVID-19 patents in their 30s and 40s in the USA [58]. Thrombotic complications in COVID-19, regardless of age, were reported by two separate scientific groups. Based on their reports, the incidence of ischemic stroke secondary to thromboembolic complications in two COVID-19 samples 
was $1.6 \%$ [59] and 2.5\% [60], respectively. As stated earlier, hemorrhagic CVAs have also been reported in COVID-19 patients [61].

Other forms of cerebrovascular pathology have been described. Acute necrotizing hemorrhagic encephalopathy (ANE) was reported in COVID-19 patients [61], with excessive cytokine release proposed as the underlying cause ("cytokine storm") [61, 62]. Poyiadji et al. [61] reported bilateral mesial temporal and thalamic hemorrhagic lesions, revealed by brain MRI in COVID-19 patients. Kremer et al. found that 16 out of 37 severely ill hospitalized COVID-19 patients had MRI signal abnormalities (nonconfluent multifocal white matter hyperintense lesions on FLAIR and diffusion sequences, with variable enhancement) in the mesio-temporal region; associated hemorrhagic lesions in 11 out of 37 patients; and extensive and isolated white matter microhemorrhages in almost a quarter of the patients. Slightly more than half (20/37) also had intracerebral hemorrhagic lesions usually associated with more severe COVID-19 presentations (ICU admission or ARDS). Somewhat surprisingly, only one patient was positive for SARS-CoV-2 RNA in CSF [63].

Pediatric immune-related COVID-19 pathology was more recently described. A number of children, in the USA and in Europe, including a 6-month-old infant, developed a Kawasaki-like syndrome, probably associated with COVID19 [64]. Kawasaki disease is characterized by a high fever and systemic vasculitis (inflammation of blood vessels), including coronary arteries and may result in neurological complications. Interestingly, as far back as 2005, Kawasaki-like syndrome was reported to be linked to a different coronavirus, HCoVNH [65]. However, the analysis of subsequent cases of this new Kawasaki-like syndrome showed clinical and laboratory features, which separate it from Kawasaki disease into a different, new clinical entity that is now called multisystem inflammatory syndrome in children (MIS-C) [66].

\section{Coronaviruses and Demyelinating IIIness}

Coronaviruses are known to cause peripheral demyelinating illness [38]. Guillain-Barre syndrome (GBS) has been described in patients with COVID-19 in a number of countries $[38,67,68]$. GBS is a form of immune-mediated peripheral neuropathy characterized by rapid-onset muscle weakness and paresthesias, when the immune system attacks the myelin sheath of peripheral nerves. Guillain-Barre symptoms do not always accompany COVID-19. However, when they do, the clinical features of GBS in COVID-19 are highly consistent: They are characterized by marked lower limb weakness over upper limb, loss of deep tendon reflexes with variable sensory abnormalities [38]. One wonders if central demyelinating illness is also possible in COVID-19 patients.
To summarize, COVID-19-related encephalopathy, as well as pathology of the peripheral NS, can be caused by multiple different mechanisms. They include direct invasion by the virus, hypoxia, and cerebrovascular events related to coagulopathy and multiple pathologies related to exaggerated/ pathological immune response. More specifically, these mechanisms/pathologies may include axonal invasion of the brain, viral crossing of blood-brain barrier, ischemic CVAs related to hypercoagulability, demyelination related to immune response, hypoxia-related encephalopathy, and cytokine-storm-related encephalopathy. There may be other, yet to be identified, mechanisms. Whether COVID-19-related neuropathologies are strictly a consequence of direct neurotropic invasion or results of indirect pathological cascades or a combination is not always clear, and a combination of several mechanisms may also be possible [38].

\section{Long-term Sequelae of COVID-19}

Conditions such as hypoxia, encephalitis, and stroke (CVA) are known to produce long-term and even permanent neurocognitive impairment. Thus, long-term neurocognitive sequelae are to be expected in a portion of COVID-19 patients after acute illness resolution. It has been anecdotally noted that COVID-19 patients who spent significant amount of time in intensive care are not infrequently left with residual cognitive impairment, sometimes referred to as "post-intensive care syndrome," a known condition, which was previously described in intensive care survivors [69]. The underlying mechanisms are unclear, but hypoxia, while on a mechanical ventilator, must be considered [70]. This possibility is supported by a recent observation of acute neuropathological changes from hypoxia in the brains of 18 consecutive deaths of ventilatordependent COVID-19 patients [71].

The possibility of long-term neurocognitive changes is particularly likely in COVID-19 patients presenting with significant neurological symptoms during the acute illness. Therefore, we may be faced with a significant number of patients with chronic and even permanent neurological and neuropsychological sequelae of COVID-19 that will require rehabilitation and present a significant societal burden in terms of health care utilization and cost and loss of productivity. Moreover, it is prudent to consider the possibility of longterm neuropsychiatric sequelae in recovered COVID-19 patients [72], especially since they have been reported in other coronavirus outbreaks (MERS) [73]. On a related note, it was reported that psychological impact of the COVID-19 pandemic is greater in psychiatric populations than in healthy controls in China [74]. This includes increased levels of anxiety, stress, depression, and insomnia. In addition, these symptoms have been identified in frontline health professionals. A recent study looking at frontline responders and health care providers 
caring for hospitalized COVID-19 patients in Wuhan, China found that $50.4 \%$ reported symptoms of depression (on PHQ9), $44.6 \%$ reported symptoms of anxiety (on GAD-7), $34 \%$ reported insomnia, and $71.5 \%$ were distressed. Rates were highest for those involved in direct care for severely ill COVID-19 patients [75]. A poignant and tragic example of such repercussions is one of the suicide by an emergency physician who worked with COVID-19 patients in New York City, NY [76].

Concerns have been also expressed about long-term mental health repercussions in the general population (even in those who did not contract COVID-19), characterized by anxiety, depression, and even suicidality [72, 77]. From a historical perspective, global disasters like the current pandemic are usually associated with an uptick of a plethora psychological/psychiatric symptoms and conditions [78]. Indeed, the number of calls to the national Disaster Distress Helpline increased by $338 \%$ in March compared with February 2020, when the current pandemic "officially" arrived in the USA. In a sample $(n=500)$ from the general Italian population, $19.4 \%$ reported mild, and $18.6 \%$ moderate-to-severe likelihood of psychological distress attributable to COVID-19 outbreak. By contrast, $62 \%$ of the sample reported no likelihood of psychological distress [79]. Significant COVID-19 psychological distress (such as symptoms of anxiety and depression) was also noted in various population segments in China [80]. The fact that these studies came out from multiple, culturally diverse countries indicates that psychological sequelae of COVID-19 are not culture specific. Addressing an extreme end of the psychological spectrum, a concern has been raised that the COVID-19 pandemic may trigger a wave of suicides [81]. A separate concern is one of long-term neurological and neurocognitive sequelae of COVID-19. In addition to acute neurological and psychological/psychiatric immediate post-acute sequelae, it has been proposed that COVID-19 survivors may be at risk for developing neurodegenerative disorders (e.g., Parkinson's disease) much later in life [82].

\section{Neuro-COVID-19}

With the discovery that coronaviruses and other respiratory viruses can invade the central nervous system (CNS) [20], the brain can be targeted by COVID-19 via multiple direct and indirect routes. As the mechanisms underlying the causes of neurological symptoms of COVID-19 are being clarified, the concept of "Neuro-COVID-19" is being increasingly embraced and the term increasingly accepted in scientific and clinical circles. A dedicated Neuro-COVID-19 unit opened at the University of Brescia Hospital in Northern Italy. There COVID-19 patients are being treated for stroke, delirium, seizures, encephalitis, and other neurological complications [83].
A research project "COVID-19 Prevalence and Cognitive Deficits in Neurological Patients (Neuro-Covid)" at Aahrus University Hospital, in Aahrus, Denmark has been initiated with 2-3 years patient follow-up expected [84]. In the UK, The CoroNerve Studies Group was created, which is a collaborative initiative to study the neurological features of COVID19. The reported purpose is to coordinate a UK nationwide surveillance program, and collaboration with international efforts [85].

\section{COVID-19 and Long-term Post-Acute Rehabilitation}

Considering all the above, high levels of physical, cognitive, and psychosocial impairments at post-acute recovery stages in a subset of COVID-19 patients can be expected. Many will require long-term rehabilitation and innovative treatment approaches [86]. Consequently, a case can be made for a longitudinal neuropsychological follow-up of recovered COVID19 patients, who exhibited neurological symptoms during the acute illness as well as of those who did not. One can draw on the experience with an HIV/AIDS epidemic. The initial understanding of HIV/AIDS was that of the virus affecting the immune system by depleting T cells, which resulted in opportunistic infections of multiple organs, including the brain. However, it did not take long to discover that the disease could also attack the brain directly, which resulted in long-term cognitive impairment. Subsequently, HIV encephalopathy and AIDS dementia complex leading to long-term cognitive impairment were discovered [87]. Based on the emerging literature, it is reasonable to hypothesize a somewhat similar scenario may unfold in relationship to COVID-19.

In support of these projections, it was reported that acute respiratory distress syndrome (ARDS) resulted in a high prevalence of long-term cognitive impairment lasting months that impacted functional status and quality of life [88]. Thus, multiple reasons for concern exist that long-term neurocognitive deficits can be expected in COVID-19 patients well after the recovery from the acute illness [89]. In response to these concerns, a survey to track long-term mental and brain health impacts of COVID-19 has been launched by neuropsychologists at the Monash University in Melbourne, Australia. They initiated a 3-year, international study to understand the mental health and cognitive effects of COVID-19 on people diagnosed with the virus, and on the general community dealing with the pandemic [90]. While we currently do not know what will be the number of post-acute COVID-19 patients requiring cognitive rehabilitation and counseling, it can be reasonably assumed that the numbers will be significant and that many COVID-19 patients, having recovered from acute illness, will require long-term follow-up for, diagnosis of, and rehabilitation of neurocognitive sequelae. 


\section{Other Coronaviruses and the Brain}

It has been previously shown that other members of the coronavirus family can affect the brain. By revisiting information that has been accumulated about their effects on the brain, one can potentially discover informative parallels and gain useful insights into what to expect from COVID-19. This, in turn, will help to guide diagnosis, treatment, and rehabilitation of COVID-19 patients.

One of the members of coronavirus family is Middle East respiratory syndrome coronavirus (MERS-CoV; MERS). MERS was first identified in 2012 in Saudi Arabia. Clinically, MERS is characterized predominantly by respiratory symptoms and a high fatality rate. Neurological manifestations, including intracerebral hemorrhagic CVAs, were reported [23]. Bickerstaff's brainstem encephalitis with Guillain-Barre syndrome features and disturbance of consciousness have been also described in MERS [19].

In a different study, neurological complications of MERS were characterized by altered levels of consciousness (ranging from confusion to coma), ataxia, and motor deficit. Several neuropsychiatric complications were also reported in MERS patients. In a sample of MERS patients in Saudi Arabia, $25.7 \%$ patients developed "insanity" and $8.6 \%$ developed seizures [23]. Brain MRI showed multiple white matter lesions in frontal, temporal, and parietal lobes, basal ganglia, and corpus callosum [24]. Post-traumatic stress disorder (PTSD) and depression in MERS survivors have also been reported. Up to 42.9\% of MERS survivors developed features of PTSD and $27.0 \%$ reported depression at 12 months following acute MERS illness [73].

Another virus from the coronavirus family has been implicated in severe acute respiratory syndrome (SARS). SARS was first recognized in the Guangdong region of China in 2002. Coronavirus (SARS-CoV) was identified as the underlying pathogen. Clinically, SARS is characterized predominantly by respiratory symptoms and a high fatality rate. Neurological manifestations, including intracerebral hemorrhagic CVA, have also been reported. SARS-CoV was shown capable of invading the brain via the olfactory bulbs with subsequent spreading among multiple brain regions, often resulting in death [18]. The fact that the axonal transport route of infection was established for other coronaviruses lends support to the theory and findings that similar mechanisms play a role in COVID-19. SARS-CoV virus particles and genome sequences could be detected in the brain, particularly in the hippocampi, yet again pointing to a direct route of infection of the brain [91]. SARS-CoV was found to be present in the cerebrospinal fluid, as well as autopsied brain tissue of SARS patients [22]. Numerous neurological complications were also reported in SARS cases including ischemic stroke [92]; neuromuscular disorders characterized by polyneuropathy and myopathy [21]; and seizures [93]. It appears that there is a significant overlap in the range of neurological symptoms/conditions across various members of the coronavirus family.

Interestingly, other coronaviruses that are usually thought to be relatively benign have been shown to be associated with Parkinson's disease (PD). Antibody levels to coronaviruses OC43 and 229E were found to be higher in the cerebrospinal fluid of PD patients than those of healthy controls [94]. An association between coronaviruses and multiple sclerosis (MS) has also been reported. Antibodies to coronaviruses OC43 and 229E were found in the cerebrospinal fluid of MS patients but not healthy controls [95]. The above studies raise the question about possible etiological or pathogenic role of coronaviruses in PD and MS. Based on these reports, an alarming question arises as to whether some of the recovered COVID-19 patients will be at a higher risk of developing neurodegenerative diseases much later in life.

Coronaviruses have been also implicated in CNS infections in children. A high level of coronavirus antibodies was found in children hospitalized for acute encephalitis-like syndrome in China [96]. Presence of human coronavirus OC43 was detected in the brain biopsy of an 11-month boy with fatal encephalitis in the UK [97]. Presence of human coronavirus was detected in the cerebrospinal fluid of a child with acute disseminated encephalomyelitis [98].

\section{Other Viruses and the Brain}

It should not come as a surprise that SARS-CoV-2 and other coronaviruses can target the brain directly, based on what we know about other viruses. Encephalopathies caused by the human immunodeficiency virus (HIV) or family of herpes viruses are examples of other human viruses that impact the brain.

It has been well established that human immunodeficiency virus (HIV) can result in an encephalopathy/encephalitis. The mechanism of this brain pathology can be a result of opportunistic infections of the brain or primary HIV encephalopathy, where HIV affects the brain directly [87]. The degree of cognitive impairment may range from very mild to full-blown AIDS dementia complex (ADC) [99]. ADC is usually seen at advanced symptomatic stages and is associated with low CD4+ T cell count. It has become increasingly rare with the advent of effective pharmacotherapies for HIV. A milder cognitive impairment, HIV-associated neurocognitive disorder (HAND), may be present already at early disease stages. Neuroimaging studies of HIV patients have found that the frontal lobes, striatum, and hippocampi are particularly impacted [100]. Cognitive impairment in HIV encephalopathy is associated with a range of symptoms, including mood and personality changes, executive deficit, memory impairment, and motor/psychomotor slowing [99]. 
The herpes simplex virus (HSV) can result in encephalopathy/encephalitis (herpes simplex encephalitis (HSE)). The usual clinical presentation is that of acute systemic illness followed by chronic cognitive impairment. The temporal lobes are particularly vulnerable [101]. This vulnerability results in cognitive deficits dominated by memory impairment. Similar but milder syndromes have been linked to cytomegalovirus (CMV), and Epstein-Barr virus (EBV) [102]. It has been suggested that HSV-1 infection is a risk factor for Alzheimer's disease in people with the epsilon4-allele of APOE gene. Risk for dementia may increase by 2.56 times in these patients with untreated HSV-1 [103, 104]. Of significant neurocognitive importance, the hippocampi, one of the anatomical structures that is crucial across many cognitive processes, is vulnerable in a number of viral encephalopathies. As discussed above, the hippocampi have been implicated as a target of viral encephalopathies in COVID-19, SARS, HSV encephalopathy, and HIV encephalopathy. Hippocampal pathology usually leads to significant memory impairment, which has important ramifications in terms of the severity of overall long-term cognitive impairment.

\section{Predicting Long-term Cognitive Sequelae of COVID-19}

Given what is known at this point about the impact of COVID-19 on the brain, the presentation of its long-term cognitive sequelae can be expected to be heterogenous in nature and will at least in part depend on the nature and severity of underlying neuropathophysiological processes at the acute COVID-19 stage. The nature of the long-term cognitive sequelae will likely be related to one of several broader categories (or a combination of categories) of potential COVID-19 neuropathological disease processes: anoxic/hypoxic/toxic encephalopathy, viral encephalitis/encephalopathy, CVA (ischemic or hemorrhagic), and demyelinating disease process (either from an immune reaction or as a result of systemic inflammatory response) $[36,43]$.

In general, chronic cognitive sequelae of anoxic/hypoxic/ toxic encephalopathy can range from subtle attention and memory impairment in mild cases to pervasive cognitive decline and dementia and severe alteration of mental state and even coma in severe cases. As in other forms of diffuse brain pathology, several broad cognitive/neurobehavioral symptoms, such as altered mental state; delirium, impaired attention/concentration, including selective attention; impaired orientation; sleep-wake cycle disturbances; and drowsiness/ lethargy can all be expected. Hypervigilance and personality changes may also develop. Retrograde amnesia, anterograde amnesia, and confabulations, poor recall can be also present, as well as neuropsychiatric symptomatology including paranoia/delusions, agitation, hallucinations, and bizarre behavior $[72,105,106]$.

Similarly, neurocognitive sequelae of COVID-19 following viral encephalitis/encephalopathy disease process may include a wide range of cognitive/neurobehavioral symptoms. However, given that COVID-19 appears, like other viral encephalopathies described above, to have particular affinity for the hippocampi [63], a severe amnestic disorder may develop against the background of less affected other cognitive functions [107]. Frontal lobes have also been mentioned as a neuroanatomical target in COVID-19 [108]; thus, executive deficit is also likely.

Cognitive sequelae of ischemic or hemorrhagic CVAs will mostly result in relatively focal neurological/cognitive deficits whose exact nature will depend on the location of the affected brain region and its size/extent. In most general terms, frontal lobe involvement may result in dysexecutive syndrome and personality/motivational changes, motor/movement impairment, apraxia, and expressive aphasia. Temporal lobe involvement can be expected to result in language and memory impairment. Parietal lobe involvement may result in the impairment of visuo-spatial functioning/perception. Occipital lobe involvement may result in impairment of visual perception and higher order visual information processing. Cerebellar involvement will result in impairment of complex motor behavior. Involvement of subcortical structures, such as basal ganglia, thalamus, hypothalamus, and amygdala, will roughly parallel impairment of their corresponding cortical projection regions and may also cause impairment of emotional processing and autonomic functions. Involvement of brain stem structures may result in overall decline of attention/ arousal and even in coma. As mentioned earlier, brain stem involvement has been hypothesized as a potential cause of respiratory problems in COVID-19 [32].

The nature and the extent of cognitive sequelae of demyelinating disease process in COVID-19 will also depend on the location of the affected white matter in the brain and its size/extent. In general, diffuse disorders of the brain white matter result in impaired neural network connectivity, which in turn manifests in sensory/motor dysfunction, impaired information processing, and slow speed of cognitive processes [109]. The overall decline in information processing impacts multiple cognitive processes, including memory, attention and concentration, executive functions, response fluency, and visuospatial functions. Extensive diffuse white matter disease of the brain can lead to dementia. More focal white matter abnormalities will result in cognitive impairment corresponding to the functions of related/adjacent cortical/subcortical neuronal structures.

In a number of patients, lasting, long-term neurocognitive impairment must be expected. Given the relatively brief history of COVID-19, one can only speculate about the specific nature of such impairment, but certain predictions can be 
made. Based on the neuroimaging data available so far, mesial temporal lobe regions appear to be particularly vulnerable in COVID-19 and are implicated more often than any other structure [49, 61]. This is likely to result in lasting memory impairment, with both anterograde and retrograde amnestic components. Brain stem has also been implicated [17, 32, 33], potentially resulting in physiological arousal impairment. Orbitofrontal involvement in COVID-19 has also been reported, potentially resulting in behavioral disinhibition and poor impulse control [110].

\section{Conclusions}

SARS-CoV-2, the virus that causes COVID-19, is one of several human viruses that affect the brain. We are only beginning to learn the exact mechanisms by, and the extent to which, it impacts the brain. Considering the rapid accumulation of knowledge about multiple neurological and neurocognitive symptoms in COVID-19 patients, NeuroCOVID-19 has been increasingly recognized as a useful clinical/scientific construct in the evolving discourse about the development of systematic approaches to the diagnosis, management, and treatment of the neurological and neurocognitive aspects of COVID-19. Many COVID-19 patients, both with and without acute CNS involvement, having recovered from acute illness, will require long-term follow-up, diagnosis, and rehabilitation of neurocognitive sequelae. They will also have to be monitored because of the potential for developing neurological disease later in life. The nature and the extent of post-COVID-19 neurocognitive sequelae will likely be vastly heterogenous in nature, depending on the underlying pathophysiological mechanisms, and will range from subtle to severe. The number of individuals suffering from long-term and even permanent cognitive deficits is likely to be significant. Multidisciplinary collaborations, involving neurology, neuropsychology, psychiatry, and physiatry, will be required in the diagnosis and treatment of COVID-19 patients in post-acute and chronic stages of the disease. In view of the sheer number of potential cases with impaired cognition and their complexity, such an effort should be, optimally, international and involve the development of a registry/ database which can be shared with and utilized by multispecialty collaborative teams of health care professionals and scientists all over the world [43]. This effort must be launched urgently and without delay.

Acknowledgments Our sincere gratitude goes to Mikhail Kazachkov, MD for his thorough review of the manuscript and helpful critique and suggestions.

Code Availability Not applicable.
Funding EG: none; DB: none; KP: funding through the John M. O'Quinn Foundation.

Data Availability Not applicable.

\section{Compliance with Ethical Standards}

Conflict of Interest The authors declare that they have no conflict of interest.

Ethics Approval Not applicable.

Consent to Participate Not applicable.

Consent for Publication Not applicable.

\section{References}

1. WHO Timeline - COVID-19. https://www.who.int/news-room/ detail/27-04-2020-who-timeline\%2D\%2D-covid-19. Accessed 22 Jun 2020

2. CORONAVIRUS RESOURCE CENTER. In: John Hopkins Univ. https://coronavirus.jhu.edu.

3. Henry R (2020) Etymologia: coronavirus. Emerg Infect Dis J 26: 1027. https://doi.org/10.3201/eid2605.et2605

4. Human coronavirus types. In: Cent. Dis. Control Prev. https:// www.cdc.gov/coronavirus/types.html. Accessed 9 Jun 2020

5. Zeng Z-Q, Chen D-H, Tan W-P, Qiu SY, Xu D, Liang HX, Chen MX, Li X et al (2018) Epidemiology and clinical characteristics of human coronaviruses OC43, 229E, NL63, and HKU1: a study of hospitalized children with acute respiratory tract infection in Guangzhou, China. Eur J Clin Microbiol Infect Dis 37:363-369. https://doi.org/10.1007/s10096-017-3144-z

6. Gupta A, Madhavan MV, Sehgal K, Nair N, Mahajan S, Sehrawat TS, Bikdeli B, Ahluwalia N et al (2020) Extrapulmonary manifestations of COVID-19. Nat Med 26:1017-1032. https://doi.org/ 10.1038/s41591-020-0968-3

7. Shang J, Wan Y, Luo C, Ye G, Geng Q, Auerbach A, Li F (2020) Cell entry mechanisms of SARS-CoV-2. Proc Natl Acad Sci U S A 117:11727-11734. https://doi.org/10.1073/pnas.2003138117

8. Natoli S, Oliveira V, Calabresi P, Maia LF, Pisani A (2020) Does SARS-Cov-2 invade the brain? Translational lessons from animal models. Eur J Neurol 27:1764-1773

9. Shang J, Ye G, Shi K, Wan Y, Luo C, Aihara H, Geng Q, Auerbach A et al (2020) Structural basis of receptor recognition by SARS-CoV-2. Nature. 581:221-224. https://doi.org/10.1038/ s41586-020-2179-y

10. Yan R, Zhang Y, Li Y et al (2020) Structural basis for the recognition of SARS-CoV-2 by full-length human ACE2. Science (80). https://doi.org/10.1126/science.abb2762

11. Butowt R, Bilinska K (2020) SARS-CoV-2: olfaction, brain infection, and the urgent need for clinical samples allowing earlier virus detection. ACS Chem Neurosci 11:1200-1203. https://doi. org/10.1021/acschemneuro.0c00172

12. Kabbani N, Olds JL (2020) Does COVID19 infect the brain? If so, smokers might be at a higher risk. Mol Pharmacol 97:351-353. https://doi.org/10.1124/molpharm.120.000014

13. Xia H, Lazartigues E (2008) Angiotensin-converting enzyme 2 in the brain: properties and future directions. J Neurochem 107: 1482-1494

14. Baig AM, Khaleeq A, Ali U, Syeda H (2020) Evidence of the COVID-19 virus targeting the CNS: tissue distribution, host- 
virus interaction, and proposed neurotropic mechanisms. ACS Chem Neurosci 11:995-998

15. Wang L, De Kloet AD, Pati D et al (2016) Increasing brain angiotensin converting enzyme 2 activity decreases anxiety-like behavior in male mice by activating central Mas receptors. Neuropharmacology. 105:114-123. https://doi.org/10.1016/j. neuropharm.2015.12.026

16. Lutchman D (2020) Could the smoking gun in the fight against Covid-19 be the (rh) ACE2? Eur Respir J 56:2001560. https://doi. org/10.1183/13993003.01560-2020

17. Li YC, Bai WZ, Hashikawa T (2020) The neuroinvasive potential of SARS-CoV2 may play a role in the respiratory failure of COVID-19 patients. J Med Virol

18. Netland J, Meyerholz DK, Moore S, Cassell M, Perlman S (2008) Severe acute respiratory syndrome coronavirus infection causes neuronal death in the absence of encephalitis in mice transgenic for human ACE2. J Virol 82:7264-7275. https://doi.org/10.1128/ jvi.00737-08

19. Kim JE, Heo JH, Kim HO, Song SH, Park SS, Park TH, Ahn JY, Kim MK et al (2017) Neurological complications during treatment of middle east respiratory syndrome. J Clin Neurol 13:227. https:// doi.org/10.3988/jen.2017.13.3.227

20. Desforges M, Le Coupanec A, Dubeau P, et al (2019) Human coronaviruses and other respiratory viruses: underestimated opportunistic pathogens of the central nervous system? Viruses

21. Tsai LK, Hsieh ST, Chao CC, Chen YC, Lin YH, Chang SC, Chang YC (2004) Neuromuscular disorders in severe acute respiratory syndrome. Arch Neurol 61:1669. https://doi.org/10.1001/ archneur.61.11.1669

22. Hung ECW, Chim SSC, Chan PKS, Tong YK, Ng EKO, Chiu RWK, Leung CB, Sung JJY et al (2003) Detection of SARS coronavirus RNA in the cerebrospinal fluid of a patient with severe acute respiratory syndrome. Clin Chem 49:2108-2109

23. Saad M, Omrani AS, Baig K, Bahloul A, Elzein F, Matin MA, Selim MAA, Mutairi MA et al (2014) Clinical aspects and outcomes of 70 patients with Middle East respiratory syndrome coronavirus infection: a single-center experience in Saudi Arabia. Int J Infect Dis 29:301-306. https://doi.org/10.1016/j.ijid.2014.09. 003

24. Arabi YM, Harthi A, Hussein J, Bouchama A, Johani S, Hajeer AH, Saeed BT, Wahbi A et al (2015) Severe neurologic syndrome associated with Middle East respiratory syndrome corona virus (MERS-CoV). Infection. 43:495-501. https://doi.org/10.1007/ s15010-015-0720-y

25. Lukiw WJ, Pogue A, Hill JM (2020) SARS-CoV-2 infectivity and neurological targets in the brain. Cell Mol Neurobiol. https://doi. org/10.1007/s10571-020-00947-7

26. Ulrich H, Pillat MM (2020) CD147 as a target for COVID-19 treatment: suggested effects of azithromycin and stem cell engagement. Stem Cell Rev Rep 16:434-440. https://doi.org/10.1007/ s12015-020-09976-7

27. Symptoms of coronavirus. In: Cent Dis Control Prev. https:// www.cdc.gov/coronavirus/2019-ncov/symptoms-testing/ symptoms.html. Accessed 9 Jun 2020

28. Politi LS, Salsano E, Grimaldi M (2020) Magnetic resonance imaging alteration of the brain in a patient with coronavirus disease 2019 (COVID-19) and anosmia. JAMA Neurol 77:1028. https:// doi.org/10.1001/jamaneurol.2020.2125

29. Wang HY, Li XL, Yan ZR et al (2020) Potential neurological symptoms of COVID-19. In: Potential neurological symptoms of COVID-19. Ther. Adv. Neurol, Disord

30. De Santis G (2020) SARS-CoV-2: a new virus but a familiar inflammation brain pattern. Brain. Behav, Immun

31. Dubé M, Le Coupanec A, Wong AHM et al (2018) Axonal transport enables neuron-to-neuron propagation of human coronavirus OC43. J Virol 92. https://doi.org/10.1128/jvi.00404-18
32. Gandhi S, Srivastava AK, Ray U, Tripathi PP (2020) Is the collapse of the respiratory center in the brain responsible for respiratory breakdown in COVID-19 patients? ACS Chem Neurosci 11: 1379-1381. https://doi.org/10.1021/acschemneuro.0c00217

33. Hsieh Y, Litvin DG, Zaylor AR et al (2020) Brainstem inflammation modulates the ventilatory pattern and its variability after acute lung injury in rodents. J Physiol 598:2791-2811. https://doi.org/ $10.1113 / \mathrm{jp} 279177$

34. Could the brain be a therapeutic target for COVID-19 patients? In: Heal. Eur. https://www.healtheuropa.eu/could-the-brain-be-atherapeutic-target-for-covid-19-patients/99841/. Accessed 9 Jun 2020

35. Xu Z, Shi L, Wang Y, Zhang J, Huang L, Zhang C, Liu S, Zhao P et al (2020) Pathological findings of COVID-19 associated with acute respiratory distress syndrome. Lancet Respir Med 8:420 422. https://doi.org/10.1016/S2213-2600(20)30076-X

36. Zanin L, Saraceno G, Panciani PP, Renisi G, Signorini L, Migliorati K, Fontanella MM (2020) SARS-CoV-2 can induce brain and spine demyelinating lesions. Acta Neurochir 162: 1491-1494. https://doi.org/10.1007/s00701-020-04374-X

37. Kandemirli SG, Dogan L, Sarikaya ZT, Kara S, Akinci C, Kaya D, Kaya Y, Yildirim D et al (2020) Brain MRI findings in patients in the intensive care unit with COVID-19 infection. Radiology. 297: E232-E235. https://doi.org/10.1148/radiol.2020201697

38. Whittaker A, Anson M, Harky A (2020) Neurological manifestations of COVID-19: a systematic review and current update. Acta Neurol Scand 142:14-22. https://doi.org/10.1111/ane.13266

39. Mao L, Jin H, Wang M, Hu Y, Chen S, He Q, Chang J, Hong C et al (2020) Neurologic manifestations of hospitalized patients with coronavirus disease 2019 in Wuhan, China. JAMA Neurol 77:683. https://doi.org/10.1001/jamaneurol.2020.1127

40. Roberts M, Levi M, Schilling R et al (2020) Covid-19: a complex multisystem clinical syndrome. BMJ Opin, In https://blogs.bmj. com/bmj/2020/05/01/covid-19-a-complex-multisystem-clinicalsyndrome/

41. Helms J, Kremer S, Merdji H, Clere-Jehl R, Schenck M, Kummerlen C, Collange O, Boulay C et al (2020) Neurologic features in severe SARS-CoV-2 infection. N Engl J Med 382: 2268-2270. https://doi.org/10.1056/nejmc2008597

42. Romero-Sánchez CM, Díaz-Maroto I, Fernández-Díaz E, Sánchez-Larsen Á, Layos-Romero A, García-García J, González E, Redondo-Peñas I et al (2020) Neurologic manifestations in hospitalized patients with COVID-19: the ALBACOVID registry. Neurology 95:e1060-e1070. https://doi.org/10.1212/WNL. 0000000000009937

43. Román GC, Spencer PS, Reis J, Buguet A, Faris MEA, Katrak SM, Láinez M, Medina MT et al (2020) The neurology of COVID-19 revisited: a proposal from the Environmental Neurology Specialty Group of the World Federation of Neurology to implement international neurological registries. J Neurol Sci 414:116884. https://doi.org/10.1016/j.jns.2020. 116884

44. Bolay H, Gül A, Baykan B (2020) COVID-19 is a real headache! Headache J Head Face Pain 60:1415-1421. https://doi.org/10. 1111/head.13856

45. Lechien JR, Chiesa-Estomba CM, De Siati DR et al (2020) Olfactory and gustatory dysfunctions as a clinical presentation of mild-to-moderate forms of the coronavirus disease (COVID-19): a multicenter European study. Eur Arch Oto-Rhino-Laryngology 277:2251-2261. https://doi.org/10.1007/s00405-020-05965-1

46. Lechien JR, Chiesa-Estomba CM, Hans S et al (2020) Loss of smell and taste in 2013 European patients with mild to moderate COVID-19. Ann Intern Med

47. Kotfis K, Williams Roberson S, Wilson JE, Dabrowski W, Pun BT, Ely EW (2020) COVID-19: ICU delirium management during SARS-CoV-2 pandemic. Crit Care 24 
48. Ye M, Ren Y, Lv T (2020) Encephalitis as a clinical manifestation of COVID-19. Brain. Behav, Immun

49. Moriguchi T, Harii N, Goto J et al (2020) A first case of meningitis/encephalitis associated with SARS-Coronavirus-2. Int J Infect Dis IJID Off Publ Int Soc Infect Dis 94:55-58

50. Haberly LB (1990) Comparative aspects of olfactory cortex. In: Jones EG, Peters A (eds) Cerebral cortex. Springer, Boston, MA

51. Vismer MS, Forcelli PA, Skopin MD et al (2015) The piriform, perirhinal, and entorhinal cortex in seizure generation. Front Neural Circuits 9:27

52. Karimi N, Sharifi Razavi A, Rouhani N (2020) Frequent convulsive seizures in an adult patient with COVID-19: a case report. Iran Red Crescent Med J 22. https://doi.org/10.5812/ircmj.102828

53. Kuroda N (2020) Epilepsy and COVID-19: associations and important considerations. Epilepsy Behav 108:107122

54. Guo YR, Cao QD, Hong ZS, et al (2020) The origin, transmission and clinical therapies on coronavirus disease 2019 (COVID-19) outbreak-an update on the status. Mil Med Res.

55. Wang Y, Wang Y, Chen Y, Qin Q (2020) Unique epidemiological and clinical features of the emerging 2019 novel coronavirus pneumonia (COVID-19) implicate special control measures. J Med Virol

56. Yanan Li (2020) Acute cerebrovascular disease following COVID-19: a single centre, retrospective, observational study. SSRN Electron $\mathrm{J}$

57. Beyrouti R, Adams ME, Benjamin L, Cohen H, Farmer SF, Goh YY, Humphries F, Jäger HR et al (2020) Characteristics of ischaemic stroke associated with COVID-19. J Neurol Neurosurg Psychiatry 91:889-891

58. Oxley TJ, Mocco J, Majidi S, Kellner CP, Shoirah H, Singh IP, de Leacy RA, Shigematsu T et al (2020) Large-vessel stroke as a presenting feature of Covid-19 in the young. N Engl J Med 382: e60

59. Klok FA, Kruip MJHA, van der Meer NJM, Arbous MS, Gommers DAMPJ, Kant KM, Kaptein FHJ, van Paassen J et al (2020) Incidence of thrombotic complications in critically ill ICU patients with COVID-19. Thromb Res 191:145-147. https://doi. org/10.1016/j.thromres.2020.04.013

60. Lodigiani C, Iapichino G, Carenzo L, Cecconi M, Ferrazzi P, Sebastian T, Kucher N, Studt JD et al (2020) Venous and arterial thromboembolic complications in COVID-19 patients admitted to an academic hospital in Milan, Italy. Thromb Res 191:9-14. https://doi.org/10.1016/j.thromres.2020.04.024

61. Poyiadji N, Shahin G, Noujaim D, Stone M, Patel S, Griffith B (2020) COVID-19-associated acute hemorrhagic necrotizing encephalopathy: CT and MRI features. Radiology. 296:E119-E120. https://doi.org/10.1148/radiol.2020201187

62. Das G, Mukherjee N, Ghosh S (2020) Neurological insights of COVID-19 pandemic. ACS Chem Neurosci 11:1206-1209

63. Kremer S, Lersy F, de Sèze J, Ferré JC, Maamar A, Carsin-Nicol $\mathrm{B}$, Collange $\mathrm{O}$, Bonneville $\mathrm{F}$ et al (2020) Brain MRI findings in severe COVID-19: a retrospective observational study. Radiology 202222:202222. https://doi.org/10.1148/radiol.2020202222

64. Jones VG, Mills M, Suarez D, Hogan CA, Yeh D, Segal JB, Nguyen EL, Barsh GR et al (2020) COVID-19 and Kawasaki disease: novel virus and novel case. Hosp Pediatr 10:537-540. https://doi.org/10.1542/hpeds.2020-0123

65. Esper F, Shapiro ED, Weibel C, Ferguson D, Landry ML, Kahn JS (2005) Association between a novel human coronavirus and Kawasaki disease. J Infect Dis 191:499-502. https://doi.org/10. $1086 / 428291$

66. Information for healthcare providers about multisystem inflammatory syndrome in children (MIS-C). In: Centers Dis. Control Prev. https://www.cdc.gov/mis-c/hcp/. Accessed 1 Jul 2020

67. Alberti P, Beretta S, Piatti M, Karantzoulis A, Piatti ML, Santoro P, Viganò M, Giovannelli G et al (2020) Guillain-Barré syndrome related to COVID-19 infection. Neurol Neuroimmunol NeuroInflammation 7:e741. https://doi.org/10.1212/NXI 0000000000000741

68. Zhao H, Shen D, Zhou H et al (2020) Guillain-Barré syndrome associated with SARS-CoV-2 infection: causality or coincidence? Neurol, Lancet

69. Needham DM, Davidson J, Cohen H, Hopkins RO, Weinert C, Wunsch H, Zawistowski C, Bemis-Dougherty A et al (2012) Improving long-term outcomes after discharge from intensive care unit: report from a stakeholders' conference. Crit Care Med 40: 502-509. https://doi.org/10.1097/CCM.0b013e318232da75

70. Ma K, Mi A, Mahmood N et al (2013) Frequency of hypoxic events in patients on a mechanical ventilator. Int J Crit Illn Inj Sci 3:124-129. https://doi.org/10.4103/2229-5151.114272

71. Solomon IH, Normandin E, Bhattacharyya S, Mukerji SS, Keller K, Ali AS, Adams G, Hornick JL et al (2020) Neuropathological features of Covid-19. N Engl J Med 383:989-992. https://oi.org/ 10.1056/nejmc2019373

72. Troyer EA, Kohn JN, Hong S (2020) Are we facing a crashing wave of neuropsychiatric sequelae of COVID-19? Neuropsychiatric symptoms and potential immunologic mechanisms. Brain Behav Immun

73. Park HY, Park WB, Lee SH, Kim JL, Lee JJ, Lee H, Shin HS (2020) Posttraumatic stress disorder and depression of survivors 12 months after the outbreak of Middle East respiratory syndrome in South Korea. BMC Public Health 20:605. https://doi.org/10. 1186/s12889-020-08726-1

74. Hao F, Tan W, Jiang L, Zhang L, Zhao X, Zou Y, Hu Y, Luo X et al (2020) Do psychiatric patients experience more psychiatric symptoms during COVID-19 pandemic and lockdown? A casecontrol study with service and research implications for immunopsychiatry. Brain Behav Immun 87:100-106. https://doi. org/10.1016/j.bbi.2020.04.069

75. Lai J, Ma S, Wang Y, Cai Z, Hu J, Wei N, Wu J, du H et al (2020) Factors associated with mental health outcomes among health care workers exposed to coronavirus disease 2019. JAMA Netw Open 3. https://doi.org/10.1001/jamanetworkopen.2020.3976

76. Watkins A, Rothfeld M, W. K. Rashbaum BMR (2020) Top E.R. doctor who treated virus patients dies by suicide. In: The NewYork Times. ttps://www.nytimes.com/2020/04/27/nyregion/ new-york-city-doctor-suicide-coronavirus.html

77. Li Z, Ge J, Yang M, Feng J, Qiao M, Jiang R, Bi J, Zhan G et al (2020) Vicarious traumatization in the general public, members, and non-members of medical teams aiding in COVID-19 control. Brain Behav Immun 88:916-919. https://doi.org/10.1016/j.bbi. 2020.03.007

78. Beaglehole B, Mulder RT, Frampton CM et al (2018) Psychological distress and psychiatric disorder after natural disasters: systematic review and meta-analysis. Br J Psychiatry

79. Moccia L, Janiri D, Pepe M, Dattoli L, Molinaro M, de Martin V, Chieffo D, Janiri L et al (2020) Affective temperament, attachment style, and the psychological impact of the COVID-19 outbreak: an early report on the Italian general population. Brain Behav Immun 87:75-79. https://doi.org/10.1016/j.bbi.2020.04.048

80. Zhang J, Lu H, Zeng H, et al (2020) The differential psychological distress of populations affected by the COVID-19 pandemic. Brain. Behav. Immun.

81. Thakur V, Jain A (2020) COVID 2019-suicides: a global psychological pandemic. Brain. Behav, Immun

82. Lippi A, Domingues R, Setz C, Outeiro TF, Krisko A (2020) SARS-CoV-2: at the crossroad between aging and neurodegeneration. Mov Disord 35:716-720

83. Talan J COVID-19: neurologists in Italy to colleagues in US: look for poorly-defined neurologic conditions in patients with the coronavirus. In: Neurol. Today 
84. Arhus U of COVID-19 prevalence and cognitive deficits in neurological patients (Neuro-Covid). In https://clinicaltrials.gov/ show/NCT04377425

85. CoroNerve. https://www.coronerve.com

86. Simpson R, Robinson L (2020) Rehabilitation following critical illness in people with COVID-19 infection. Am J Phys Med Rehabil:1. https://doi.org/10.1097/PHM.0000000000001443

87. Cecilia RE, Ovidiu R, Mihaela S, Dana CR (2012) HIV-associated neurocognitive disorders: a historical review. Neurologist 18:64 67

88. Sasannejad C, Ely EW, Lahiri S (2019) Long-term cognitive impairment after acute respiratory distress syndrome: a review of clinical impact and pathophysiological mechanisms. Crit Care 23

89. Li J, Long X, Zhang Q, Fang X, Fang F, Lv X, Zhang D, Sun Y et al (2020) Emerging evidence for neuropsycho-consequences of COVID-19. Curr Neuropharmacol 18. https://doi.org/10.2174/ 1570159x18666200507085335

90. University M Survey to track long-term mental, brain health impacts of COVID-19. In: Monash Univ. https://www.monash.edu/ news/articles/survey-to-track-long-term-mental,-brain-healthimpacts-of-covid-19

91. Gu J, Gong E, Zhang B, Zheng J, Gao Z, Zhong Y, Zou W, Zhan J et al (2005) Multiple organ infection and the pathogenesis of SARS. J Exp Med 202:415-424. https://doi.org/10.1084/jem. 20050828

92. Tsai LK, Hsieh ST, Chang YC (2005) Neurological manifestations in severe acute respiratory syndrome. Acta Neurol Taiwanica

93. Ng Kee Kwong KC, Mehta PR, Shukla G, Mehta AR (2020) COVID-19, SARS and MERS: a neurological perspective. J Clin Neurosci 77:13-16. https://doi.org/10.1016/j.jocn.2020.04. 124

94. Fazzini E, Fleming J, Fahn S (1992) Cerebrospinal fluid antibodies to coronavirus in patients with Parkinson's disease. Mov Disord 7:153-158. https://doi.org/10.1002/mds.870070210

95. Salmi A, Ziola B, Hovi T, Reunanen M (1982) Antibodies to coronaviruses OC43 and $229 \mathrm{E}$ in multiple sclerosis patients. Neurology. 32:292. https://doi.org/10.1212/wnl.32.3.292

96. Li Y, Li H, Fan R, Wen B, Zhang J, Cao X, Wang C, Song Z et al (2017) Coronavirus infections in the central nervous system and respiratory tract show distinct features in hospitalized children. Intervirology. 59:163-169. https://doi.org/10.1159/000453066

97. Morfopoulou S, Brown JR, Davies EG, Anderson G, Virasami A, Qasim W, Chong WK, Hubank M et al (2016) Human coronavirus OC43 associated with fatal encephalitis. N Engl J Med 375: 497-498

98. Yeh EA, Collins A, Cohen ME et al (2004) Detection of coronavirus in the central nervous system of a child with acute disseminated encephalomyelitis. Pediatrics. 113:e73-e76. https:// doi.org/10.1542/peds.113.1.e73

99. Alford K, Banerjee S, Nixon E, O'Brien C, Pounds O, Butler A, Elphick C, Henshaw P et al (2019) Assessment and management of HIV-associated cognitive impairment: experience from a multidisciplinary memory service for people living with HIV. Brain Sci 9. https://doi.org/10.3390/brainsci9020037

100. Heyes MP (2001) Elevated cerebrospinal fluid quinolinic acid levels are associated with region-specific cerebral volume loss in HIV infection. Brain. 124:1033-1042. https://doi.org/10.1093/ brain/124.5.1033

101. Whitley RJ (2006) Herpes simplex encephalitis: adolescents and adults. Antivir Res 71:141-148

102. Eeg-Olofsson O (2003) Virological and immunological aspects of seizure disorders. Brain and Development 25:9-13

103. Middleton PJ, Petric M, Kozak M, Rewcastle NB, Crapper McLachlan DR (1980) Herpes-simplex viral genome and senile and presenile dementias of Alzheimer and pick. Lancet 315:1038

104. Tzeng NS, Chung CH, Lin FH, Chiang CP, Yeh CB, Huang SY, Lu RB, Chang HA et al (2018) Anti-herpetic medications and reduced risk of dementia in patients with herpes simplex virus infections - a nationwide, population-based cohort study in Taiwan. Neurotherapeutics. 15:417-429. https://doi.org/10.1007/ s13311-018-0611-x

105. Shprecher D, Mehta L (2010) The syndrome of delayed posthypoxic leukoencephalopathy. NeuroRehabilitation 26:65-72. https://doi.org/10.3233/NRE-2010-0536

106. Anderson CA, Arciniegas DB (2010) Cognitive sequelae of hypoxic-ischemic brain injury: a review. NeuroRehabilitation 26:47-63. https://doi.org/10.3233/NRE-2010-0535

107. Scolville WB, Milner B (1957) Loss of recent memory after bilateral hippocampal lesions. J Neurol Neurosurg Psychiatry 20:1121. https://doi.org/10.1136/jnnp.20.1.11

108. Paniz-Mondolfi A, Bryce C, Grimes Z, Gordon RE, Reidy J, Lednicky J, Sordillo EM, Fowkes M (2020) Central nervous system involvement by severe acute respiratory syndrome coronavirus-2 (SARS-CoV-2). J Med Virol 92:699-702

109. Sumowski JF, Benedict R, Enzinger C et al (2018) Cognition in multiple sclerosis: state of the field and priorities for the future. Neurology. https://doi.org/10.1212/WNL.0000000000004977

110. Le Guennec L, Devianne J, Jalin L et al (2020) Orbitofrontal involvement in a neuroCOVID-19 patient. Epilepsia. 61. https:// doi.org/10.1111/epi.16612

Publisher's Note Springer Nature remains neutral with regard to jurisdictional claims in published maps and institutional affiliations. 